\title{
High expression of piwi-like RNA-mediated gene silencing 1 is associated with poor prognosis via regulating transforming growth factor- $\beta$ receptors and cyclin-dependent kinases in breast cancer
}

\author{
JIWEI CAO*, GANG XU* , JING LAN, QINGQING HUANG, ZUXIONG TANG and LIPING TIAN \\ Department of General Surgery, The First Affiliated Hospital of Soochow University, Suzhou, Jiangsu 215000, P.R. China
}

Received October 21, 2014; Accepted October 19, 2015

DOI: $10.3892 / \mathrm{mmr} .2016 .4842$

\begin{abstract}
Previous studies have demonstrated that abnormal expression levels of PIWI may serve a crucial role in tumorigenesis. However, the pathological role and its association with prognosis remains to be fully elucidated. In the present study, the expression levels of piwi-like RNA-mediated gene silencing 1 (HIWI) and piwi-like RNA-mediated gene silencing 2 (HILI) in breast cancer tissues were reported to be high. The high expression levels of HIWI are correlated with poor prognosis in detected patients. In addition, by overexpression and interference, it was demonstrated that HIWI promotes the activity of breast cancer cells while depression of HIWI may induce apoptosis of breast cancer cells. It was additionally identified that suppression of HIWI may arrest the cells at the $\mathrm{G}_{2} / \mathrm{M}$ stage. The expression levels of transforming growth factor- $\beta$ receptor (T $\beta R) I, T \beta R I I$, cyclin-dependent kinase (CDK)4, CDK6 and CDK8 were observed to be regulated by HIWI, which indicated a novel mechanism of HIWI in the regulation of breast cancer progression. The present study provides novel insight into the HIWI expression in breast cancer, providing a potential biomarker for assessment of prognosis and therapy of breast cancer.
\end{abstract}

\section{Introduction}

Breast cancer is one of most common causes of cancer-associated mortality worldwide (1). In general, the development stage of breast cancer includes the benign proliferation, precancer and cancer like other types of cancer (1). During the early stages of breast cancer, intervention of the progression of the precancer

Correspondence to: Professor Liping Tian, Department of General Surgery, The First Affiliated Hospital of Soochow University, 188 Shizi Road, Suzhou, Jiangsu 215000, P.R. China

E-mail: lptian88@126.com

${ }^{*}$ Contributed equally

Key words: HIWI, breast cancer, T $\beta$ R, CDK, prognosis can eradicate the cancer. Once organisms have entered into the cancer stages, breast cancer has a poor survival rate (3). Thus, accurate and effective specific biomarkers for breast cancer are required for monitoring the progression of breast cancer. At present, the commonly used therapeutic strategies for breast cancer are surgical resection, chemotherapy and radiotherapy (3). However, these treatments have a low long-term survival rate (1). The pathogenesis of cancer is complex, involving genetic, epigenetic and environmental factors $(4,5)$. Thus, improvement in the understanding of the developmental mechanisms would aid in the diagnosis of patients and the development of effective treatment strategies. Studies investigating the epigenetics of cancer may provide a strategy for the identification of diagnostic biomarkers and for novel therapeutic strategies.

In cancer cells, the genomes are often unstable, exhibiting a duplication and/or deletion of genes and deviant epigenetic alterations (5). Previously, epigenetic alterations including mRNA silencing, genome methylation and histone modification during the progression of cancer have been thoroughly investigated $(5,6)$. Genome hypomethylation influences cancer progression by affecting transcription and transposable elements (TEs) (7). Several previous studies have indicated that the methylation levels of oncogenes and tumor suppressor genes are associated with abnormal expression of these genes, and result in increased cancer progression (5-9). In addition, the unstable TEs in genome transfer in the chromosomes have been reported to lead to deleterious mutations and aberrant expression of oncogenes and tumor suppressor genes $(4,10)$. These active TEs contribute to the progression of cancer. Thus, epigenetics may potentially be used for diagnosis and monitoring of prognosis. However, the association between epigenetics and cancer progression remains unclear.

In previous studies, a broad pathway, the PIWI-piRNA pathway, was identified in germline cells from mouse testes $(11,12)$. Subsequent studies demonstrated that this pathway was highly conserved in different organisms, from invertebrates to mammals (13-15). PIWI homologs were initially suggested to be specifically expressed in germline cells. Low and high expression levels of PIWI have been reported to result in reproductive disorders in different animals, including those in flies (14), fish (15), frogs (16) and 
mammals (17). Previously, studies have identified aberrant expression levels of PIWI in multiple types of human cancer, including ovarian, lung and pancreatic cancer $(18,19)$. In breast, endometrial and gastrointestinal tract cancer, PIWI expression has been detected, while in normal tissues, the expression of PIWI was not observed (20). Thus, it is suggested that PIWI participates in the progression of cancer. In humans, four different homologs, including piwi-like RNA-mediated gene silencing 1 (PIWIL1/HIWI), piwi-like RNA-mediated gene silencing 2 (PIWIL2/HILI), piwi-like RNA-mediated gene silencing 3 (PIWIL3) and piwi-like RNA-mediated gene silencing 4 (PIWIL4/HIWI2) have been identified (13). The function of these different homologs remains to be fully elucidated. HIWI has been regarded as an inducer for tumor growth and mortality (21). The expression of HILI has been identified in different types of cancer cells, including those of breast and cervical cancer (19). PIWIL3 and HIWI2 have been identified in colon cancer alone (22). Thus, the expression profiles and its association with cancer progression require further investigation.

The present study focused upon the expression profile of PIWI homologs during the progression of breast cancer, and in association with the patient prognosis. In addition, by transfection of cells, the effects of overexpression, interference, overexpression reversal and interference reversal of the key PIWI homologs were assayed in the breast cancer cell line MCF-7. Furthermore, the role of HIWI in regulating the expression levels of T $\beta R$ and cyclin-dependent kinases (CDKs) was investigated in the breast cancer cell line MCF-7.

\section{Materials and methods}

Materials. The human tissue samples including normal $(\mathrm{n}=25)$, benign $(n=19)$ and malignant $(n=8)$ tissues were obtained from 27 female patients (age, 30-45 years) during tumor resection at the First Hospital Affiliated to Soochow University (Suzhou, China). The tissue type was confirmed pathologically. All patients provided written informed consent and approval was provided by the Ethics Committee of the First Hospital Affiliated to Soochow University. Informed consent was obtained from all patients.

Reverse transcription-quantitative polymerase chain reaction $(R T-q P C R)$. The gene expression in tissues was assayed by RT-qPCR. The total RNAs of the tissue samples were extracted by TRIzol reagent (Invitrogen Life Technologies, Carlsbad, CA, USA) according to the manufacturer's instructions. Subsequently, $1 \mu \mathrm{g}$ RNA was transcribed to the first-strand cDNA using SuperScript II reverse transcriptase (Invitrogen Life Technologies). The primers used are presented in Table I. $\beta$-actin was used as an internal control. RT-qPCR was performed with the Applied Biosystems 7500 Real-Time PCR System (Applied Biosystems Life Technologies, Foster City, CA, USA). The PCR conditions were as follows: $94^{\circ} \mathrm{C}$ for 2 min followed by 40 cycles of $94^{\circ} \mathrm{C}$ for $15 \mathrm{sec}$ and $60^{\circ} \mathrm{C}$ for $45 \mathrm{sec}$. A final extension step was conducted at $72^{\circ} \mathrm{C}$ for $30 \mathrm{sec}$. Subsequent to the amplification, melting curve analysis was conducted to confirm the specificity of the amplification products using AB 7500 software, version 2.0.6 (Applied Biosystems; Thermo Fisher Scientific, Inc., Waltham, MA,
Table I. Primer sequences for reverse transcription-quantitative polymerase chain reaction.

\begin{tabular}{|c|c|}
\hline Gene & Primer sequence $5^{\prime} \rightarrow 3^{\prime}$ \\
\hline MAEL & F: CTGATGATAGAACCAGAGTC \\
\hline MAEL & R: GAATCCAAGTCTTAGAGGGC \\
\hline HIWI & F: GAAGCAGCCTGTCTTGGTCAGCCAGCCTG \\
\hline HIWI & R: GAATCAAAGCTCAAACCCCAGTCTC \\
\hline HILI & F: ATCTATATCTGGCTGCTCCTC \\
\hline HILI & R: GATGCAAGATGTGTCCTGAC \\
\hline PIWIL3 & F: GGTGATTTGTATCCTGCCCA \\
\hline PIWIL3 & R: TGACCATCTCCCACTCCATC \\
\hline HIWI2 & F: AATGCTCGCTTTGAACTAGAGAC \\
\hline HIWI2 & R: ATTTTGGGGTAGTCCACATTAAATC \\
\hline T $\beta R I$ & F: TGGCGGGGAGAAGAAGTTG \\
\hline $\mathrm{T} \beta \mathrm{RI}$ & R: CTGCTGCTATAAATCCCAGGATG \\
\hline T $\beta R I I$ & F: AGAAGTCGGATGTGGAAATGGA \\
\hline $\mathrm{T} \beta \mathrm{RII}$ & R: CTGCACCGTTGTTGTCAGTG \\
\hline CDK4 & F: CAGGACCTAAGGACATATCTGGA \\
\hline CDK4 & R: CTCGGTACCAGAGTGTAACAACC \\
\hline CDK6 & F: TGATCAACTAGGAAAAATCTTGGAC \\
\hline CDK6 & R: GGCAACATCTCTAGGCCAGT \\
\hline CDK8 & F: ATCAGTCGGGCTGGTGCTG \\
\hline CDK8 & R: CTTTATCATCCTTCCCATCTTTCC \\
\hline$\beta$-actin & F: CACCATGAAGATCAAGATCATTGC \\
\hline$\beta$-actin & R: GGCCGGACTCATCGTACTCCTGC \\
\hline
\end{tabular}

MAEL, maelstrom spermatogenic transposon silencer; HIWI, piwi-like RNA-mediated gene silencing 1; HILI, piwi-like RNA-mediated gene silencing 2; PIWIL3, piwi-like RNA-mediated gene silencing 3; HIWI2, piwi-like RNA-mediated gene silencing 4; T $\beta R I$, transforming growth factor- $\beta$ type I receptor; CDK, cyclin-dependent kinase; $F$, forward; $R$, reverse.

USA). For each sample, RT-qPCR reactions were performed in triplicate. The results were analyzed with the $2^{-\Delta \Delta C t}$ method (23).

Western blot analysis. The tissues were lysed in radioimmunoprecipitation assay buffer (Beyotime Institute of Biotechnology, Haimen, China) on ice and centrifuged at $12,000 \mathrm{xg}$ for $30 \mathrm{~min}$ at $4^{\circ} \mathrm{C}$ to obtain the protein. The extracted proteins were then separated by $15 \%$ polyacrylamide gels and transferred onto polyvinylidene difluoride membranes (GE Healthcare Life Sciences, New Orleans, LA, USA). Subsequent to blocking in milk for $1 \mathrm{~h}$, the membranes were incubated with the dilution of primary antibodies overnight at $4^{\circ} \mathrm{C}$. The primary antibodies were: Rabbit polyclonal anti-human MAEL (1:500; Abcam, Cambridge, MA, USA; cat. no. ab106713), rabbit polyclonal anti-human HIWI (1:500; Abcam; cat. no. ab12337), rabbit polyclonal anti-human HILI (1:500; Abcam; cat. no. ab181340), rabbit polyclonal anti-human PIWIL3 (1:500; Abcam; cat. no. ab93709), rabbit polyclonal anti-human HIWI2 (1:500; Abcam; cat. no. 180867) and rabbit polyclonal anti-human GAPDH (1:2,000; Abcam; cat. no. ab9485). The samples were washed with phosphate-buffered saline (PBS; Beyotime 
Institute of Biotechnology) three times and then probed with the goat anti-rabbit horseradish peroxidase (HRP)-conjugated IgG $(\mathrm{H}+\mathrm{L})$ secondary antibody $(1: 2,000$; Thermo Fisher Scientific, Inc.; cat. no. 31460) for $1 \mathrm{~h}$ at room temperature. The signals were detected using the Bio-Rad ChemiDoc Touch System (Bio-Rad Laboratories, Inc., Hercules, CA, USA). Three independent experiments were repeated, with each sample protein normalized to GAPDH.

Cell culture. The MCF-7 cell line was provided by the American Type Culture Collection (Manassas, VA, USA). These cells were incubated in Dulbecco's modified Eagle's medium (DMEM; Gibco Life Technologies, Grand Island, NY, USA), which was supplemented with $10 \%$ fetal bovine serum (Invitrogen Life Technologies), at $37^{\circ} \mathrm{C}$ in an incubator with $5 \% \mathrm{CO}_{2}$. The viability of the cells was analyzed using trypan blue exclusion (Sangon Biotech Co., Ltd., Shanghai, China) subsequent to cell culture for $24 \mathrm{~h}$. For the detection of cellular morphology, the cells were grown in glass cover slips for $24 \mathrm{~h}$ at $37^{\circ} \mathrm{C}$ and observed using an inverted microscope (PM-10AD; Olympus Corporation, Tokyo, Japan).

Cell transfection. The interference and overexpression vectors were constructed in order to examine the biophysical properties of the cell line subsequent to knockdown and overexpression of HIWI. The transfection vector was constructed using a plasmid of pcDNA3.1(t) (pc3.1) (Invitrogen Life Technologies) and HIWI. The open reading frame fragment of HIWI was obtained and cloned into pc3.1 between the BamHI and EcoRI sites in order to construct the recombinant plasmids. Subsequent to construction of the plasmids, transfection was conducted using Lipofectamine ${ }^{\mathrm{TM}} 2000$ (Invitrogen Life Technologies) according to the manufacturer's instructions. The cells were cultured in DMEM at $37^{\circ} \mathrm{C}$ in an incubator and collected at $24 \mathrm{~h}$ for the following analysis.

The cells were divided into five groups (5 parallel treatments for each group), including the control (non-treated group), HIWI interference group (1 $\mu \mathrm{g}$ HIWI shRNA plasmid transfection), HIWI overexpression group (1 $\mu \mathrm{g}$ pcDNA3.1(t)-HIWI plasmid transfection), HIWI interference reversal group (1 $\mu \mathrm{g}$ HIWI shRNA plasmid transfection for $12 \mathrm{~h}$ followed by $1 \mu \mathrm{g}$ pcDNA3.1(t)-HIWI plasmid transfection for $12 \mathrm{~h})$ and HIWI overexpression reversal group $(1 \mu \mathrm{g}$ pcDNA3.1(t)-HIWI plasmid transfection for $12 \mathrm{~h}$ followed by $1 \mu \mathrm{g}$ HIWI shRNA plasmid transfection for $12 \mathrm{~h}$ ).

Flow cytometry. Flow cytometric analysis was used to investigate the apoptosis of the cells. All fluorescence signals of labeled cells were detected using the FACScan flow cytometer (BD Biosciences, San Jose, CA, USA). Using the Annexin V-Fluorescein Isothiocyanate (FITC) Apoptosis Detection kit (BD Biosciences), the phosphatidylserine redistribution in the plasma membranes was measured according to the manufacturer's instructions.

Statistical analysis. All data are presented as the mean \pm standard error. The statistical analyses were conducted using SPSS software, version 17.0 (SPSS, Inc., Chicago, IL, USA). The significant differences among the groups were determined by one-way analysis of variance. The Cox's regression analysis
Table II. Characteristics for the 187 patients at the beginning of the experiment.

\begin{tabular}{lccc}
\hline & \multicolumn{2}{c}{ Expression } & \\
\cline { 2 - 3 } Characteristic & Low $(\mathrm{n}=98)$ & High $(\mathrm{n}=89)$ & P-value \\
\hline Age & $47.98 \pm 5.62$ & $49.52 \pm 7.84$ & 0.58 \\
Stage I & 32 & 41 & 0.45 \\
Stage II & 48 & 37 & 0.78 \\
Stage III + IV & 18 & 11 & 0.74 \\
Tumor size $\left(\mathrm{mm}^{3}\right)$ & $5.42 \pm 2.61$ & $23.54 \pm 1.62$ & 0.012 \\
\hline
\end{tabular}

based on gene expression and prognostic outcome was performed. The follow-up information for 187 patients for a period of 100 months was collected. The patients were divided into two groups: higher HIWI expression group (mRNA relative expression, $>5$ ) and the lower HIWI expression group (mRNA relative expression, $>2$ but $<4$ ). The result was calculated by fitting the predictive prognosis model using SPSS. In addition, expression of HIWI and HILI were analyzed using a Kruskal-Wallis test, followed by Dunn's multiple comparison tests with SPSS. P<0.05 was considered to indicate a statistically significant difference.

\section{Results}

Expression levels of HIWI and HILI are increased in malignant breast cancer tissues. In order to investigate the important role of PIWI in breast cancer, RT-qPCR and western blotting were conducted in order to analyze the expression of HIWI, HILI, PIWIL3, HIWI2 and maelstrom spermatogenic transposon silencer (MAEL) in normal breast tissue $(n=25)$, benign breast tumors $(n=19)$ and malignant breast cancer tumors $(n=8)$. The results demonstrated that the relative expression levels of MAEL, HIWI and HILI were significantly higher in malignant breast cancer tissues compared with normal and benign breast tissues (Fig. 1A-C). However, the relative expression levels of PIWIL3 and HIWI2 in malignant breast tissues were not significantly different to the normal and benign breast tissues (Fig. 1D and E). Furthermore, the results of western blotting of these genes were consistent with the results of RT-qPCR (Fig. 1F). The protein expression levels of MAEL, HIWI and HILI in malignant breast cancer were significantly higher than those of normal and benign breast tissues, while no significant differences in PIWIL3 and HIWI2 expression levels were observed between the three tissue groups.

HIWI is associated with a reduced prognosis in breast cancer. The follow-up information for 187 patients for a period of 100 months was collected (Table II). The patients were first divided into two groups: The higher HIWI expression group (mRNA relative expression $>5$ ) and the lower HIWI expression group (mRNA relative expression $>2$ ). The tumor specific survival rates with HIWI and HILI are presented in Fig. 2A. For HIWI, a significant correlation between HIWI and survival rate was identified $(\mathrm{P}<0.05)$. The higher HIWI expression group exhibited significantly higher survival curves than the 

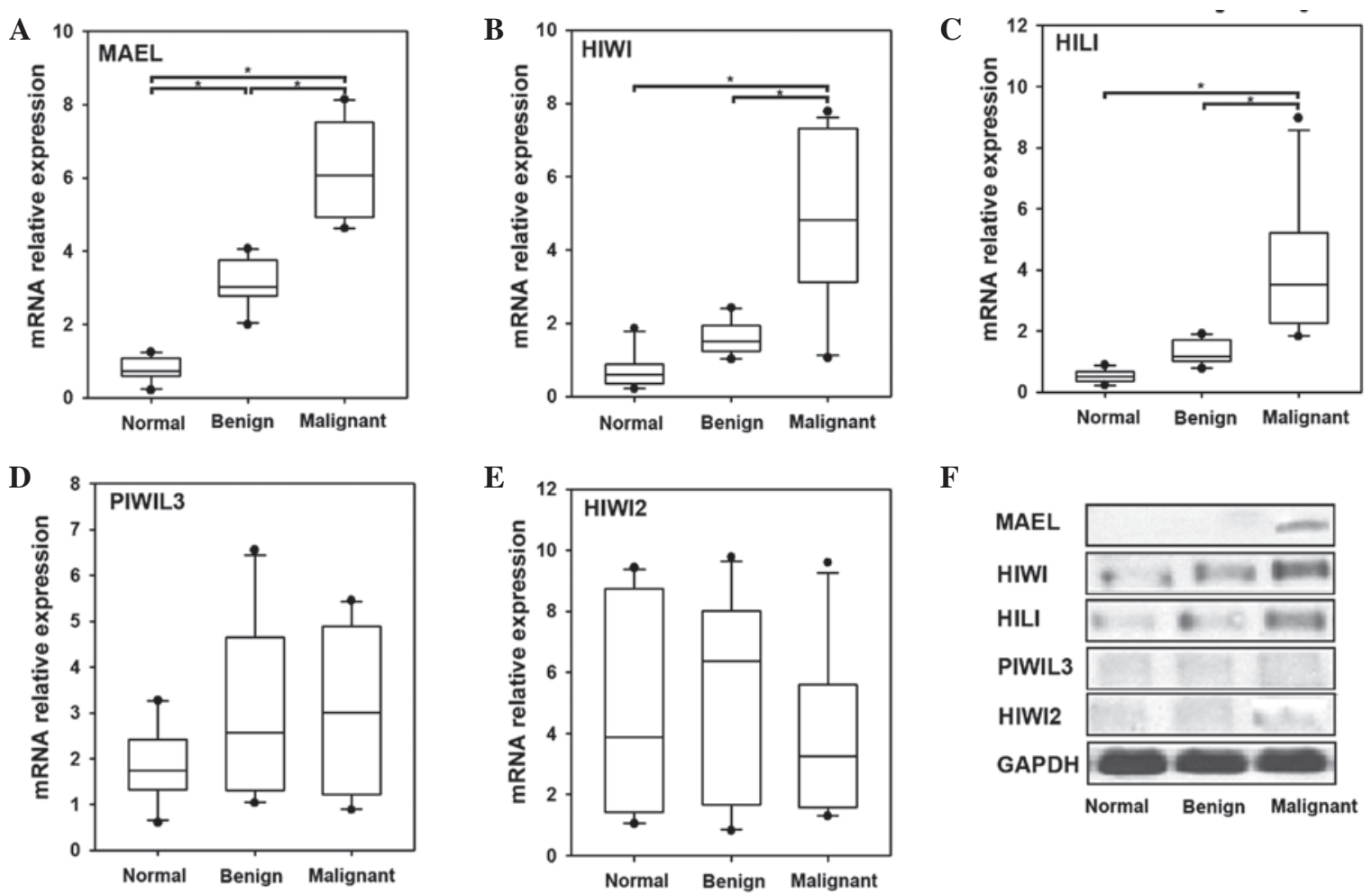

F

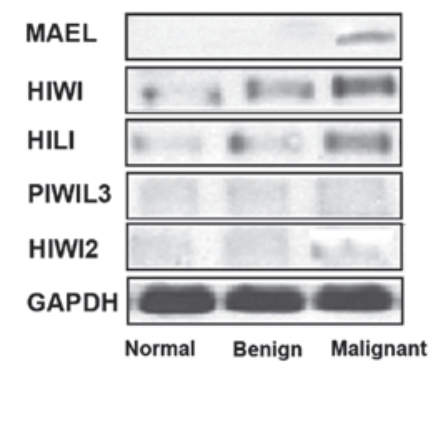

Figure 1. Box plots representing the expression of HIWI pathway genes in normal breast, benign breast cancer and malignant breast cancer tissues. Reverse transcription-quantitative polymerase chain reaction analysis of mRNA expression of (A) MAEL, (B) HIWI, (C) HILI, (D) PIWIL3 and (E) HIWI2. (F) The protein expression levels of HIWI pathway genes in normal breast, benign breast cancer and malignant breast cancer tissues. The Kruskal-Wallis test was performed to compare the gene expression levels between the two groups. Filled dots indicate the mean of each group, ${ }^{*} \mathrm{P}<0.05$. HIWI, piwi-like RNA-mediated gene silencing 1; MAEL, maelstrom spermatogenic transposon silencer; HILI, piwi-like RNA-mediated gene silencing 2; PIWIL3, piwi-like RNA-mediated gene silencing 3; HIWI2, piwi-like RNA-mediated gene silencing 4.

A

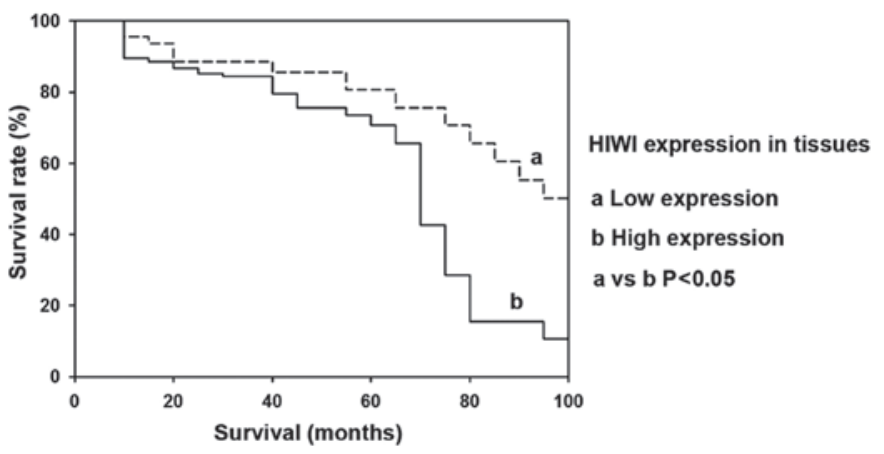

B

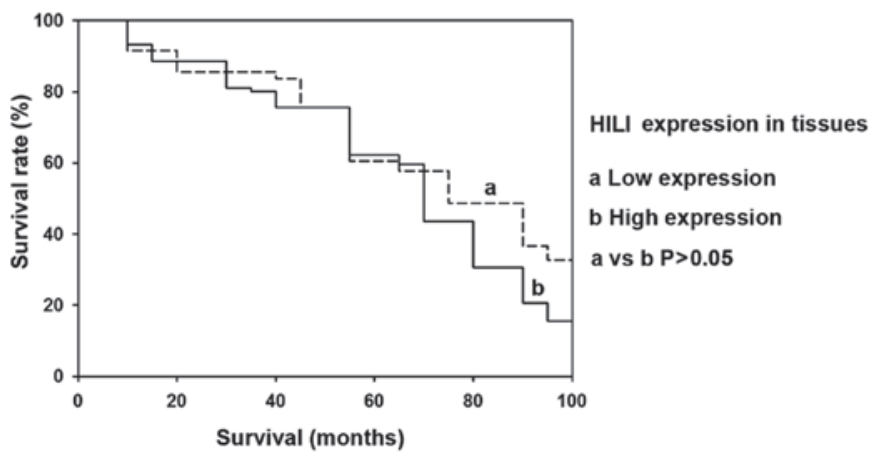

Figure 2. Multivariate Cox's regression analyses. (A) Expression of HIWI and its correlation with tumor-specific survival. (B) Expression of HILI and its correlation with tumor-specific survival. HIWI, piwi-like RNA-mediated gene silencing 1; HILI, piwi-like RNA-mediated gene silencing 2.

lower HIWI expression group. However, no significant differences were observed for HILI (Fig. 2B).

Regulation of breast cancer cell progression by HIWI. In order to detect the effect of HIWI on breast cancer cell lines, the interference and overexpression HIWI vectors were constructed and transfected into cells by Lipofectamine ${ }^{\mathrm{TM}}$ 2000. The mRNA expression and protein expression was analyzed by RT-qPCR and western blotting, respectively.

The results indicated that the highest expression of HIWI was present in the overexpression group and the lowest mRNA expression was observed in the interference group. While the control group, overexpression reversal group and interference reversal group exhibited intermediate expression (Fig. 3A). Accordingly, the protein expression results were similar to that of the mRNA expression. The overexpression group had the strongest signals compared with the other groups. The interference group had the lowest expression of HIWI protein among all of the groups (Fig. 3B).

To elucidate the effects of HIWI on cellular regulation, the cell cycle progression and cellular apoptosis were detected by flow cytometry. The results demonstrated that the lowest apoptotic rate in was in the overexpression group, whereas the highest apoptotic rate was in the interference group (Fig. 3C 
A

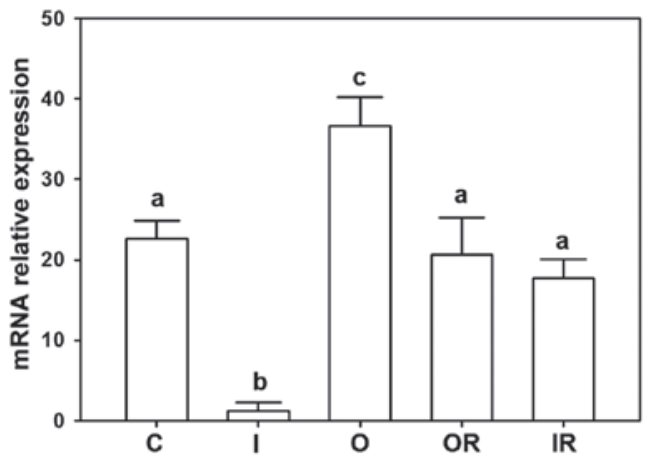

C

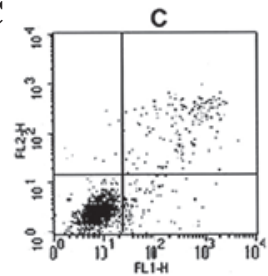

D

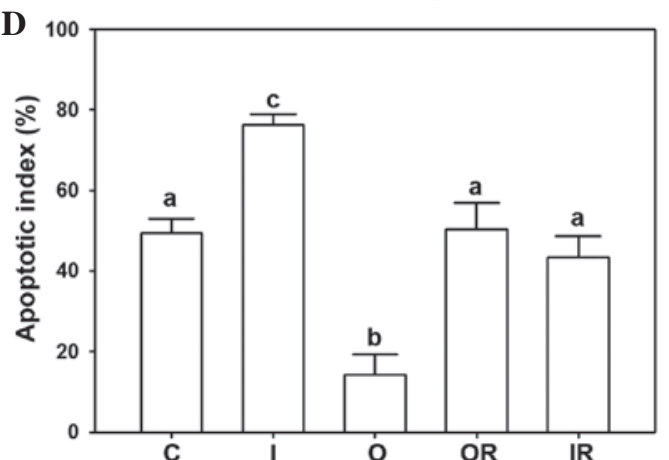

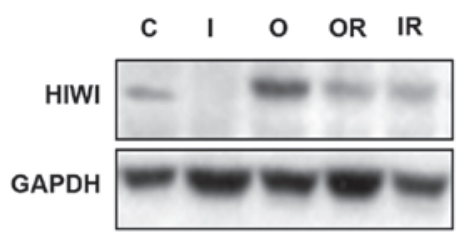
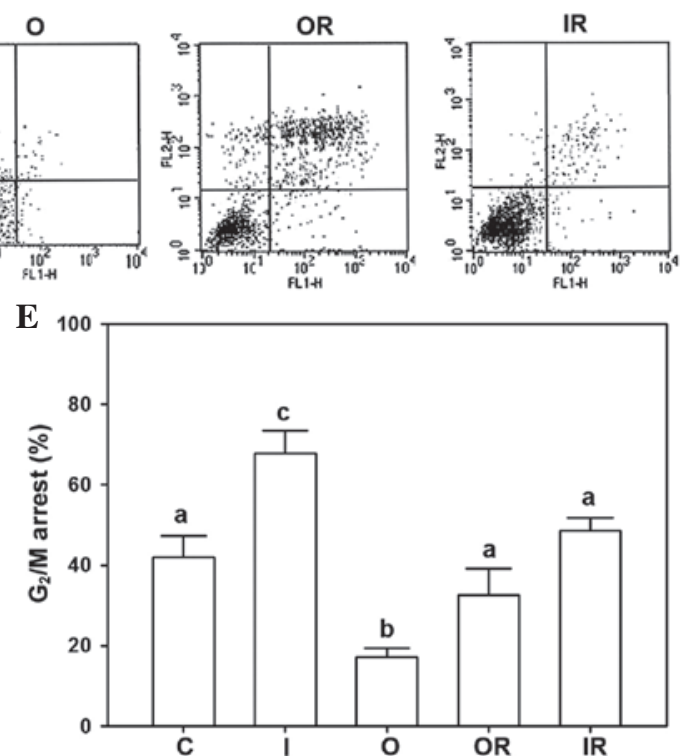

Figure 3. Interference and overexpression of HIWI in breast cancer cells. (A) HIWI mRNA expression analyzed by reverse transcription-quantitative polymerase chain reaction. (B) HIWI protein expression analyzed by western blotting. (C) Apoptosis analysis by flow cytometry among all the treatment groups. (D) Apoptotic index and (E) $\mathrm{G}_{2} / \mathrm{M}$ arrested rate inferred from flow cytometric analysis. HIWI, piwi-like RNA-mediated gene silencing 1; C; control; I, interference; O, overexpression; OR, overexpression reversal; IR, interference reversal.
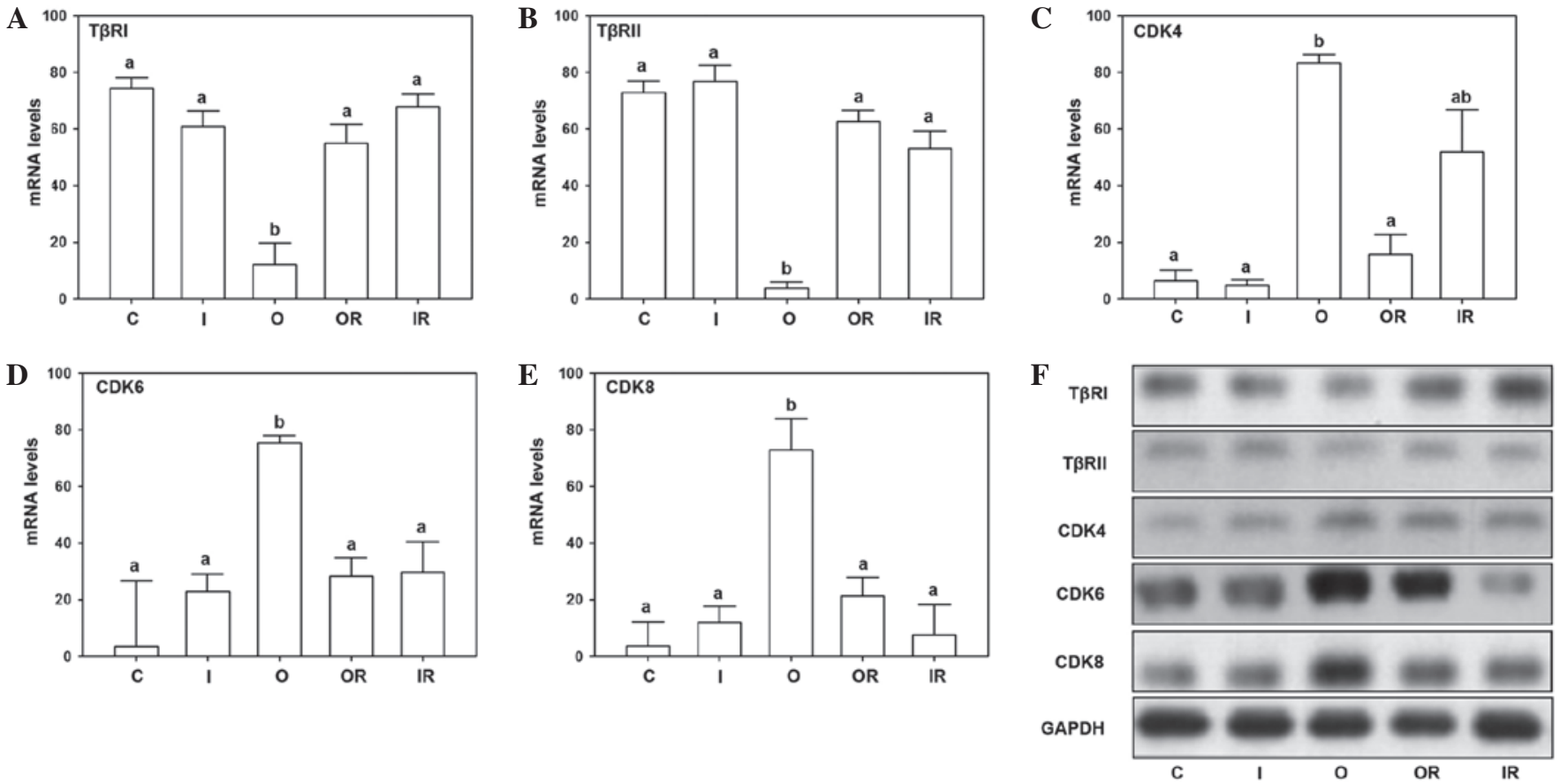

Figure 4. HIWI regulates T $\beta R$ and CDKs in the breast cancer cell line. mRNA expression of (A) T $\beta R I,(B)$ T $\beta$ RII, (C) CDK4, (D) CDK6 and (E) CDK8 analyzed by reverse transcription-quantitative polymerase chain reaction (characters indicate a significant difference; $\mathrm{P}<0.05$ ). (F) Protein expression levels of T $\beta R$ I, T $\beta$ RII, CDK4, CDK6 and CDK8 analyzed by western blotting. HIWI, piwi-like RNA-mediated gene silencing 1; CDK, cyclin-dependent kinase; T $\beta R I$, transforming growth factor- $\beta$ type I receptor; $\mathrm{C}$, control; I, interference; O, overexpression; OR, overexpression reversal; IR, interference reversal. 
and D). In addition, the interference group exhibited a significantly greater rate of $\mathrm{G}_{2} / \mathrm{M}$ arrest compared with other groups (Fig. 3E).

HIWI regulates T $\beta R$ and $C D K$ expression levels in breast cancer cells. In order to demonstrate the mechanism of HIWI regulation in the T $\beta R$ and CDK pathway, the mRNA and protein expression levels of T $\beta R$ and CDK were assayed. The mRNA and protein expression of T $\beta R I$ and $T \beta R I I$ were observed to be downregulated by overexpression of HIWI, while the other groups had no significant differences (Fig. 4A, B and F). However, the mRNA and protein expression levels of CDK4 were upregulated by overexpression of HIWI (Fig. 4C). The mRNA expression levels of CDK4 were the lowest in the control, interference and overexpression reversal groups, with no significant differences observed (Fig. 4C). While the expression in the interference reversal group exhibited no significant differences from the other groups. By contrast, the levels of CDK6 and CDK8 in the overexpression group exhibited the highest expression, while no significant differences were observed in the remaining four groups (Fig. 4D and E). In addition, CDK4, CDK6 and CDK8 exhibited a significant increase in the overexpression group, while other groups exhibited no significant differences (Fig. 4F).

\section{Discussion}

In the present study, it was demonstrated for the first time, to the best of our knowledge, that overexpression of PIWI promotes progression of breast cancer in vivo and in vitro. By comparing the expression levels of PIWI genes among normal, benign and malignant tissues, it was demonstrated that HIWI and HILI exhibited high levels of expression in malignant breast cancer. The results indicated that these two overexpressed genes may mediate the progression of breast cancer. Furthermore, it was identified that high expression levels of HIWI are associated with an impaired prognosis, while HILI did not exhibit such effects in breast cancer. Previous studies have demonstrated similar results, observing high expression levels of PIWI genes in colorectal and endometrial cancer $(13,24,25)$. In these types of cancer, the overexpression of PIWI was observed to be associated with the initiation and progression of cancer. In addition, PIWI homologs have been observed to exhibit varied alterations in different types of cancer studied. For example, HILI is highly expressed in precancerous stem cells during tumorigenesis (19) while in colon cancer, only PIWIL3 and HIWI2 have been observed $(22,26)$. In breast cancer, Liu et al (20) demonstrated that HILI was expressed in various stages of cancer, and they proposed that HILI may be a potential biomarker for diagnosis. The results of the current study additionally suggested that high expression of HILI is present in malignant breast cancer, however the association of patient prognosis with HILI was poor. By contrast, high expression of HIWI was additionally observed in malignant breast cancer, and a significant correlation between HIWI expression and prognosis was identified. Thus, based on the observations of the current study, HIWI is suggested as a more suitable biomarker for breast cancer than HILI.

Subsequent to identification of the abnormally high expression of HIWI during the development of breast cancer, the question of how this factor influences the progression of cancer was addressed. Thus, transfection was used to detect the effect of HIWI on progression of breast cancer cells. Subsequent to interference and overexpression of HIWI in cells, the expression levels were altered accordingly. The flow cytometry results suggested that overexpression of HIWI promoted cell activity and inhibited the apoptosis of cells compared with the control group. Subsequent to interference of HIWI, the apoptotic rate increased significantly. Activities of tumor cells, including metastasis and invasion, are regulated by the cell cycle (27). Previous studies have demonstrated that HIWI participates in the stability of the genome and regulates genes expression via binding with piRNAs $(7,28)$. The PIWI-piRNA pathway was only identified in 2006, thus the understanding of this pathway in cancer remains limited (29). Although HIWI is aberrantly expressed in human cancer and its correlation with prognosis has been reported in previous studies $(21,30,31)$, the effects of HIWI on the activities of cells remain unclear. In addition, interference of HIWI may inhibit the progression of breast cancer cells, which provides a novel potential therapy for use in cancer treatment.

HILI may inhibit transforming growth factor $\beta$ (TGF- $\beta$ ) signaling by regulating $\mathrm{Hsp} 90$ and promoting T $\beta \mathrm{R}$ degradation (32). In the present study, the results suggested that HIWI promoted cell activities. Thus, the current study additionally investigated gene regulation by HIWI. The results suggested that inhibition of HIWI arrested cells in the $\mathrm{G}_{2} / \mathrm{M}$ stage. Due to the fact that HIWI may regulate cell progression of breast cancer via controlling the cell cycle, the cell cycles were affected by HIWI. The results suggest that TGF- $\beta$ signaling and/or CDK genes may be responsible for cell cycle regulation via HIWI. The results of the current study demonstrated that overexpression of HIWI reduced the levels of T $\beta$ RI and T $\beta$ RII, which is similar to the result of HILI overexpression. However, it remains unclear whether this effect is mediated by HILI. In addition, the expression of HIWI, which is suggested to regulate $\mathrm{T} \beta \mathrm{R}$ degradation, may serve a crucial role in the progression of cancer. In order to understand the molecular mechanism of HIWI on the cell cycle, the expression levels of factors associated with the cell cycle, including CDK4, CDK6 and CDK8 were detected (33). The results demonstrated that overexpression of HIWI promotes mRNA in addition to protein expression levels of these genes. The members of the CDK family serve an important role in the cell cycle (34). In the CDK family, CDK4, CDK6 and CDK8 are key genes which control DNA synthesis and the transition from $\mathrm{G}_{1}-\mathrm{S}$ stage (33). Under or overexpression of these genes leads to dysfunction of cell progression (34). Thus, it is proposed that the overexpression of HIWI in breast cancer contributed to the promotion of CDK gene expression and the induction of cancer progression.

In conclusion, the present study demonstrated that high expression levels of HIWI are associated with a reduction in the patient prognosis in breast cancer. Suppression of HIWI may arrest the cells at the $\mathrm{G}_{2} / \mathrm{M}$ stage. Furthermore, the expression levels of T $\beta$ RI, T $\beta$ RII, CDK4, CDK6 and CDK8 were regulated by HIWI in breast cancer cells. These observations indicate that HIWI participates in the progression of breast cancer via regulating the gene expression of $\mathrm{T} \beta \mathrm{R}$ s and CDKs. 


\section{References}

1. Ferlay J, Soerjomataram I, Dikshit R, Eser S, Mathers C, Rebelo M, Parkin DM, Forman D and Bray F: Cancer incidence and mortality worldwide: Sources, methods and major patterns in GLOBOCAN 2012. Int J Cancer 136: E359-E386, 2015.

2. Montagna E, Cancello G, Dellapasqua S, Munzone E and Colleoni M: Metronomic therapy and breast cancer: A systematic review. Cancer Treat Rev 40: 942-950, 2014.

3. Snee M: Follow-up of women treated for breast cancer. Clin Oncol (R Coll Radiol) 8: 85-89, 1996.

4. Nowsheen S, Aziz K, Tran PT, Gorgoulis VG, Yang ES and Georgakilas AG: Epigenetic inactivation of DNA repair in breast cancer. Cancer Lett 342: 213-222, 2014.

5. Fucito A, Lucchetti C, Giordano A and Romano G: Genetic and epigenetic alterations in breast cancer: What are the perspectives for clinical practice? Int J Biochem Cell Biol 40: 565-575, 2008.

6. Sandoval $\mathrm{J}$ and Esteller M: Cancer epigenomics: Beyond genomics. Curr Opin Genet Dev 22: 50-55, 2012.

7. Chénais B: Transposable elements and human cancer: A causal relationship? Biochim Biophys Acta 1835: 28-35, 2013.

8. Hansen KD, Timp W, Bravo HC, Sabunciyan S, Langmead B, McDonald OG, Wen B, Wu H, Liu Y, Diep D, et al: Increased methylation variation in epigenetic domains across cancer types. Nat Genet 43: 768-775, 2011.

9. Lopez-Serra P and Esteller M: DNA methylation-associated silencing of tumor-suppressor microRNAs in cancer. Oncogene 31: 1609-1622, 2012.

10. Levin HL and Moran JV: Dynamic interactions between transposable elements and their hosts. Nat Rev Genet 12: 615-627, 2011.

11. Girard A, Sachidanandam R, Hannon GJ and Carmell MA: A germline specific class of small RNAs binds mammalian Piwi proteins. Nature 442: 199-202, 2006.

12. Grivna ST, Beyret E, Wang Z and Lin H: A novel class of small RNAs in mouse spermatogenic cells. Genes Dev 20: 1709-1714, 2006.

13. Seto AG, Kingston RE and Lau NC: The coming of age for Piwi proteins. Mol Cell 26: 603-609, 2007.

14. Senti KA and Brennecke J: The piRNA pathway: A fly's perspective on the guardian of the genome. Trend Genet 26 : 499-509, 2010.

15. Zhou Y, Zhong H, Liu S, Yu F, Hu J, Zhang C, Tao M and Liu Y: Elevated expression of Piwi and piRNAs in ovaries of triploid crucian carp. Mol Cell Endocrinol 383: 1-9, 2014.

16. Zhang D, Duarte-Guterman P, Langlois VS and Trudeau VL: Temporal expression and steroidal regulation of piRNA pathway genes (mael, piwi, vasa) during Silurana (Xenopus) tropicalis embryogenesis and early larval development. Comp Biochem Physiol C Toxicol Pharmacol 152: 202-206, 2010.

17. Xu M, You Y, Hunsicker P, Hori T, Small C, Griswold MD and Hecht NB: Mice deficient for a small cluster of Piwi-interacting RNAs implicate Piwi-interacting RNAs in transposon control. Biol Reprod 79: 51-57, 2008
18. Ye Y, Yin DT, Chen L, Zhou Q, Shen R, He G, Yan Q, Tong Z, Issekutz AC, Shapiro CL, et al: Identification of Piwil2-like (PL2L) proteins that promote tumorigenesis. PLoS One 5: e13406, 2010.

19. Nikpour P, Forouzandeh-Moghaddam M, Ziaee SA, Dokun OY, Schulz WA and Mowla SJ: Absence of PIWIL2 (HILI) expression in human bladder cancer cell lines and tissues. Cancer Epidemiol 33: 271-275, 2009.

20. Liu JJ, Shen R, Chen L, Ye Y, He G, Hua K, Jarjoura D, Nakano T, Ramesh GK, Shapiro CL, et al: Piwil2 is expressed in various stages of breast cancers and has the potential to be used as a novel biomarker. Int J Clin Exp Pathol 3: 328-337, 2010.

21. Taubert H, Greither T, Kaushal D, Würl P, Bache M, Bartel F, Kehlen A, Lautenschläger C, Harris L, Kraemer K, et al: Expression of the stem cell self-renewal gene Hiwi and risk of tumour-related death in patients with soft-tissue sarcoma. Oncogene 26: 1098-1100, 2007.

22. Li L, Yu C, Gao H and Li Y: Argonaute proteins: Potential biomarkers for human colon cancer. BMC Cancer 10: 38, 2010.

23. Livak KJ and Schmittgen TD: Analysis of relative gene expression data using real-time quantitative PCR and the 2(-Delta Delta C(T)) method. Methods 25: 402-408, 2001.

24. Liu W, Jiang X and Zhang Z: Expression of PSCA, PIWIL1, and TBX2 in endometrial adenocarcinoma. Oncol Res Treat 33: 241-245, 2010.

25. Oh SJ, Kim SM, Kim YO and Chang HK: Clinicopathologic implications of PIWIL2 expression in colorectal cancer. Korean J Pathol 46: 318-323, 2012.

26. Samowitz WS: Genetic and epigenetic changes in colon cancer. Exp Mol Pathol 85: 64-67, 2008.

27. Liang D, Fang Z, Dong M, Liang C, Xing C, Zhao J and Yang Y: Effect of RNA interference-related HiWi gene expression on the proliferation and apoptosis of lung cancer stem cells. Oncol Lett 4: 146-150, 2012.

28. O'Donnell KA and Boeke JD: Mighty Piwis defend the germline against genome intruders. Cell 129: 37-44, 2007.

29. Siddiqi S and Matushansky I: Piwis and piwi-interacting RNAs in the epigenetics of cancer. J Cell Biochem 113: 373-380, 2012.

30. Grochola LF, Greither T, Taubert H, Möller P, Knippschild U, Udelnow A, Henne-Bruns D and Würl P: The stem cell-associated Hiwi gene in human adenocarcinoma of the pancreas: Expression and risk of tumour-related death. Br J Cancer 99: 1083-1088, 2008.

31. Liu X, Sun Y, Guo J, Ma H, Li J, Dong B, Jin G, Zhang J, Wu J, Meng L, et al: Expression of hiwi gene in human gastric cancer was associated with proliferation of cancer cells. Int J Cancer 118: 1922-1929, 2006.

32. Zhang K, Lu Y, Yang P, Li C, Sun H, Tao D, Liu Y, Zhang S and Ma Y: HILI inhibits TGF- $\beta$ signaling by interacting with Hsp90 and promoting T $\beta R$ degradation. PLoS One 7: e41973, 2012.

33. Shah MA and Schwartz GK: Cyclin-dependent kinases as targets for cancer therapy. Update Cancer Ther 1: 311-332, 2006.

34. Graña X and Reddy EP: Cell cycle control in mammalian cells: Role of cyclins, cyclin dependent kinases (CDKs), growth suppressor genes and cyclin-dependent kinase inhibitors (CDKIs). Oncogene 11: 211-219, 1995. 